\title{
Lesson of the month 2: Fever, arthralgias and hyperkeratotic, scaling and fissuring eruptions of the hands
}

\author{
Authors: Evangelia Zampeli ${ }^{A}$ and Haralampos M Moutsopoulos ${ }^{B}$
}

\begin{abstract}
A 56-year-old male hairdresser, presented with fever and arthralgias. He had a worsening 'eczematous' palmar rash which had been attributed to irritation from colouring products used in his work. On examination, hyperkeratotic, fissuring eruptions were noted on the palmar side of his fingers and lung auscultation revealed crackles on both lower lobes. Laboratory testing showed elevated muscle enzymes. The clue to the diagnosis in our patient was the presence of hyperkeratotic, fissuring eruptions on the palmar side of his fingers, compatible with 'mechanic's hands'. This is a characteristic semiology reminiscent of hands of manual workers, resembling hand eczema. 'Mechanic's hands', fever, arthralgias, lung findings and elevated serum muscle enzymes raised the possibility of antisynthetase syndrome. Testing for myositis autoantibodies revealed anti-Jo-1 and anti-Ro52 reactivity supporting the diagnosis.

Greater awareness of the typical clinical feature of 'mechanic's hands' will allow for earlier diagnosis and appropriate treatment in patients with antisynthetase syndrome.
\end{abstract}

KEYWORDS: Mechanic's hands, myositis, idiopathic inflammatory myopathies, antisynthetase syndrome

\section{Case Description}

A previously healthy 56 -year-old man, hairdresser in profession, presented with a 1-month history of low-grade fever, increased fatigue and knee arthralgias. A progressively worsening 'eczematous', fissuring and scaling rash on the palmar side of his fingers had also developed. These manifestations were attributed to irritation from colouring products used in his work. A week before presentation he noted also some difficulty in swallowing solid food.

On examination, vital signs and oxygen saturation on ambient air were normal. Palmar skin was rough, thickened and nonpruritic. Hyperkeratotic, scaling and fissuring eruptions were

Authors: A rheumatologist, Institute for Autoimmune Systemic and Neurological Diseases, Athens, Greece; ${ }^{B}$ emeritus professor, Institute for Autoimmune Systemic and Neurological Diseases, Athens, Greece and Academy of Athens, Athens, Greece noted mainly on the palmar side of his index fingers and thumbs (Fig 1). A widespread, flat redness was evident on his upper back and shoulders. Lung auscultation revealed crackles on both lower lobes. The rest of the physical examination, including evaluation of muscle strength of upper and lower limbs and neurologic examination, was normal. Laboratory testing was remarkable for elevated serum creatine phosphokinase $814 \mathrm{U} / \mathrm{L}$ (normal range $(\mathrm{nr}$ ) = 22-198 U/L), lactate dehydrogenase $705 \mathrm{U} / \mathrm{L}(\mathrm{nr}=100-190 \mathrm{U} / \mathrm{L})$, aspartate aminotransferase $60 \mathrm{U} / \mathrm{L}(\mathrm{nr}=10-40 \mathrm{U} / \mathrm{L})$ and positive antinuclear antibodies (1:640 cytoplasmic pattern).

\section{Discussion}

The clue to the correct diagnosis in our patient was the presence of hyperkeratotic, scaling and fissuring eruptions on the palmar side of the index fingers and thumbs, compatible with 'mechanic's hands'. This is a characteristic semiology of the palmar skin reminiscent of hands of manual workers, resembling hand eczema. Hyperkeratosis involving the ulnar aspect of the thumb and the radial aspect of index/middle fingers is typical. ${ }^{1}$ Recently it has been proposed that patients with 'mechanic's hands' have similar findings on their soles ('hiker's feet'). ${ }^{2}$ A detailed medical history and physical examination will differentiate hand eczema from 'mechanic's hands'. Lesions are symmetrical, not related to the dominant hand and pruritus is absent. ${ }^{3}$ In our patient, 'mechanic's hands' along with fever, arthralgias, mild dysphagia, crackles on lung auscultation and elevated serum muscle enzymes raised the possibility of antisynthetase syndrome (ASS). ${ }^{4}$ Lung computed tomography showed lower lobe ground-glass attenuation bilaterally. Testing for myositis-specific and-associated autoantibodies revealed anti-Jo-1 and anti-Ro52 reactivity supporting the diagnosis of ASS.

ASS is a clinically distinguishable idiopathic inflammatory myopathy (IIM) subset characterised by fever, non-erosive arthritis, Raynaud's phenomenon, 'mechanic's hands', interstitial lung disease and myositis, along with circulating antisynthetase antibodies. ${ }^{4}$ ASS prognosis depends predominantly on lung involvement. $^{5}$

In ASS patients' serum, autoantibodies directed against eight different aminoacyl-tRNA synthetases (ARS) can be found. Those are the anti-Jo-1/(histidyl), anti-PL-7/(threonyl), anti-PL-12/(alanyl), anti-OJ/(isoleucyl), anti-EJ/(glycyl), anti-KS/(asparaginyl), anti-Zo/ (phenylalanyl) and anti-Ha/(tyrosyl). Anti-Jo-1 is the hallmark of ASS, found in $70-80 \%$ of patients. ${ }^{6,7}$ In IIM patients as a whole, 
Fig 1. Hyperkeratotic, scaling and fissuring eruptions on the patient's palmar side of the index fingers and thumbs.

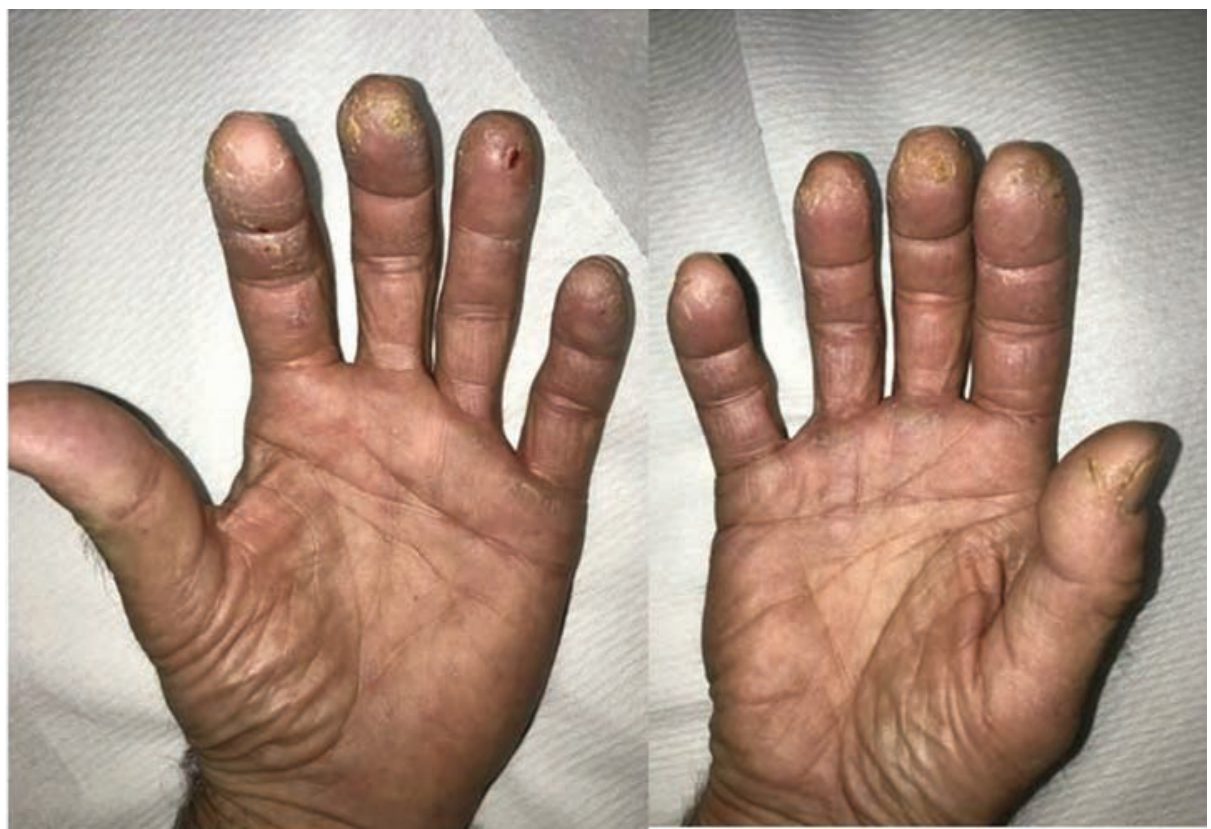

anti-Jo1 is detected in $20-30 \%$ of them, while the other ARS autoantibodies are infrequent $(5 \%)^{6,7}$

Myositis in ASS is often absent or subclinical; therefore muscle biopsy is not typically performed. Nevertheless, the occurrence of ex-novo clinical manifestations during follow-up is typical for ASS patients. ${ }^{5,8}$ Histology from an affected muscle in ASS patients will show prominent perimysial connective tissue fragmentation, and perifascicular necrosis. ${ }^{9}$ ASS, as all IIMs, is treated with immunosuppressives. Corticosteroids have long been the first-line treatment for ASS. Adjunctive immunosuppressives (azathioprine, mycophenolate mofetil, tacrolimus/cyclosporine, rituximab, and cyclophosphamide) are frequently used. These are added in refractory cases and/or as corticosteroid-sparing agents, ${ }^{10}$ yet patients with ASS often require multi-modality immunosuppressive therapy.

Our patient received monthly intravenous pulses of methylprednisolone (1 gm) with cyclophosphamide $\left(1 \mathrm{gm} / \mathrm{m}^{2}\right.$ body surface area) for six months with a good response. The long-term follow-up of ASS patients mandates monitoring of ILD progression and possible immunosuppressive therapy adverse effects.

It is hoped that greater awareness of the typical clinical feature of 'mechanic's hands' will allow for earlier diagnosis and appropriate treatment to improve outcomes in patients with ASS.

\section{References}

1 Sohara E, Saraya T, Sato $S$ et al. Mechanic's hands revisited: is this sign still useful for diagnosis in patients with lung involvement of collagen vascular diseases? BMC Res Notes 2014;17:303.
2 Chen WCW, Baily JE, Corselli M et al. 'Hiker's feet': a novel cutaneous finding in the inflammatory myopathies. Clin Rheumatol 2017:36:1683-6.

3 Bygum A, Velander MJ, Knudsen JB. Mechanic's hands imitating hand eczema. Dermatitis 2010;21:334-5.

4 Mahler M, Miller FW, Fritzler MJ. Idiopathic inflammatory myopathies and the anti-synthetase syndrome: A comprehensive review. Autoimmun Rev 2014;13:367-71.

5 Bartoloni E, Gonzalez-gay MA, Scirè C et al. Clinical follow-up predictors of disease pattern change in anti-Jo1 positive antisynthetase syndrome : Results from a multicenter, international and retrospective study. Autoimmun Rev 2017;16:253-7.

6 Brouwer R, Hengstman GJD, Vree Egberts W et al. Autoantibody profiles in the sera of European patients with myositis. Ann Rheum Dis 2001;60:116-23.

7 Zampeli E, Venetsanopoulou A, Argyropoulou OD et al. Myositis autoantibody profiles and their clinical associations in Greek patients with inflammatory myopathies. Clin Rheumatol 2019:38:125-32.

8 Cavagna L, Nuño L, Scirè CA et al. Clinical spectrum time course in anti jo-1 positive antisynthetase syndrome: Results from an international retrospective multicenter study. Medicine (Baltimore) 2015;94:e1144.

9 Dalakas MC. Inflammatory muscle diseases. N Engl J Med 2015;372:1734-47.

10 Witt LJ, Curran J], Strek ME. The diagnosis and treatment of antisynthetase syndrome. Clin Pulm Med 2016;23:218-26.

Address for correspondence: Dr Evangelia Zampeli, Institute for Autoimmune Systemic and Neurological Diseases, M Asias 75, 11527, Athens, Greece Email:zampelieva@gmail.com 